\title{
Alternative Knochenanalyse bei Neugeborenen - Quantitativer Ultraschall des peripheren Skeletts
}

\section{Bone Analysis in the Newborn - Quantitative Ultrasound of the Peripheral Skeleton}

Autoren

Elena Basemann ${ }^{1}$, Kristin Dawczynski², Hans Proquitté ${ }^{2}$, Axel Hübler ${ }^{3}$, Hans-Joachim Mentzel ${ }^{1}$

Institute

1 Sektion Kinderradiologie, Institut für Diagnostische und Interventionelle Radiologie, Universitätsklinikum Jena

2 Sektion Neonatologie und Pädiatrische Intensivmedizin, Universitätsklinikum Jena

3 Klinik für Kinder- und Jugendmedizin, Klinikum Chemnitz gGmbH, Chemnitz

Schlüsselwörter

Quantitativer Ultraschall, Osteopenie, Frühgeborene, Neugeborene, Referenzwerte, Osteoporose

Key words

Quantitative ultrasound, osteopenia, osteoporosis, preterm babies, newborn, reference values

eingereicht

24.02.2019

angenommen nach Überarbeitung

18.04.2019

Bibliografie

DOI https://doi.org/10.1055/a-0915-9828

Online-Publikation: 24.6.2019

Z Geburtsh Neonatol 2020; 224: 79-85

(c) Georg Thieme Verlag KG Stuttgart · New York

ISSN 0948-2393

Korrespondenzadresse

Prof. Dr. Hans-Joachim Mentzel

Institut für Diagnostische und Interventionelle Radiologie,

Sektion Kinderradiologie

Universitätsklinikum Jena

07747 Jena

hans-joachim.mentzel@med.uni-jena.de

\section{ZUSAMMENFASSUNC}

Ziel Bestimmung des quantitativen Ultraschallparameters Schallgeschwindigkeit (Speed of Sound, SOS) bei Neugeborenen zur Erhebung von Referenzdaten und Untersuchung von Einflussfaktoren.
Material und Methoden 219 Neu- und 14 Frühgeborene (28. - 41. Schwangerschaftswoche) mit Geburtsgewicht zwischen 590 und $4930 \mathrm{~g}$ wurden in den ersten Lebenstagen mit Sunlight Omnisense 7000 P untersucht. Referenzdaten für SOS wurden bei 178 eutrophen Neugeborenen an der Tibia erhoben.

Ergebnisse SOS-Referenzwerte an der Tibia liegen bei $3028 \pm 92 \mathrm{~m} / \mathrm{s}<40$. SSW und $3057 \pm 96 \mathrm{~m} / \mathrm{s} \geq 40$. SSW. Zwischen SOS und Gestationsalter besteht eine signifikante Korrelation, männliche Neugeborene weisen signifikant höhere SOS auf. Bei Geburtsgewicht $<3800 \mathrm{~g}$ besteht eine Korrelation $z$ wischen SOS und Gewicht $(p<0,05)$. Frühgeborene haben signifikant niedrigere SOS als eutrophe Neugeborene $(p<0,05)$. Schlussfolgerungen Der Ultraschallparameter SOS kann bei Früh- und Neugeborenen suffizient an der Tibia ermittelt werden. Die erhobenen Referenzdaten von kaukasischen Neugeborenen sind geeignet, um eine Frühgeborenen-Osteopenie zu identifizieren.

\section{ABSTRACT}

Purpose The aim was to determine whether the quantitative ultrasound (QUS) parameter speed of sound (SOS) is sufficient for evaluation of reference data in the newborn and how they are influenced.

Materials and Methods 219 full-term and 14 preterm infants (gestational age 28-41 weeks (GA), birth weight 590-4,930 g) were evaluated by Omnisense $7000 \mathrm{P}$ on their tibia. Reference values were sampled in 178 eutrophic infants.

Results SOS reference values at the tibia were $3028 \mathrm{~m} / \mathrm{s}$ for $\mathrm{GA}<40$ and $3057 \mathrm{~m} / \mathrm{s}$ for $\geq 40$ weeks. There was a significant correlation between SOS and gestational age. SOS was significantly higher in male $(3058.3 \pm 93.3$ vs. $3022.8 \pm 93.4 \mathrm{~m} / \mathrm{s} ; \mathrm{p}$ 0.012 ) infants. If birth weight was below $3,800 \mathrm{~g}$, there was a correlation between SOS and weight $(p<0.05)$. Preterm infants showed lower SOS than term babies $(p<0.05)$.

Conclusion Speed of sound can be evaluated sufficiently in preterm and term infants on the tibia. The estimated reference data can be used to identify osteopenia in the preterm infant with low birth weight. 


\section{Einleitung}

Die Osteoporose - gekennzeichnet durch geringe Knochenmasse und erhöhtes Frakturrisiko - stellt ein weltweit bedeutendes gesundheitspolitisches Problem dar. Da 80 \% der Mineralsalzeinlagerung in den fetalen Knochen während des letzten Trimesters der Schwangerschaft erfolgen, ist es nicht verwunderlich, dass 39\% der Frühgeborenen mit einem Geburtsgewicht von unter $1500 \mathrm{~g}$ eine Osteopenie aufweisen [1]. Nach der Geburt erfolgt beim Reifgeborenen physiologisch infolge veränderter hormoneller und nutritiver Versorgung sowie geringer mechanischer Stimulation innerhalb der ersten 6 Lebensmonate ein Abfall der physikalischen Knochendichte, vorrangig im Bereich der langen Röhrenknochen bei gleichzeitiger Zunahme von Kortikalisdicke und Mineralgehalt. Von dieser physiologischen Veränderung abgegrenzt werden muss die pathologische Osteopenie des Früh-/Neugeborenen, für die es neben der Unreife verschiedene andere Faktoren (z. B. unzureichende Versorgung mit Mineralsalzen, verminderte Absorption, Darmpathologien, renale Verluste, Pathologie des Vitamin-D-Metabolismus) geben kann [2]. Um das Ausmaß einer Osteopenie bei Frühgeborenen zu ermitteln, werden im klinischen Alltag die Serumwerte für Kalzium, Phosphat und Alkalische Phosphatase und die Urinwerte für Kalzium und Phosphat genutzt. Diese Laborchemie reflektiert zwar den Knochenmineralumsatz, korreliert aber nicht mit der tatsächlichen Knochendichte oder mechanischen Elastizität und Stabilität des Knochens [3].

Die Osteoporosediagnostik und Verlaufsbeurteilung wird nach Empfehlung des Dachverbands Osteologie mittels Zwei-SpektrenRöntgenabsorptiometrie (Dual energy X-ray absorptiometry, DXA) durchgeführt, mit der die Knochenmineraldichte (bone mineral density = BMD) abgeschätzt werden kann [4]. Nachteile sind neben der Strahlenexposition (mit ca. $1 \mu$ Sv sehr gering) und dem hohen apparativen Aufwand v. a. der Charakter als planare Messmethode, mit der die Masse pro Fläche $\left(\mathrm{g} / \mathrm{cm}^{2}\right)$ und nicht die Dichte $(\mathrm{g} /$ $\mathrm{cm}^{3}$ ) ermittelt wird. Bei Neugeborenen konnte zwischen der DXABMD und dem Geburtsgewicht sowie dem Gestationsalter ein Zusammenhang nachgewiesen werden. Frühgeborene weisen niedrigere Werte auf. Sie zeigen im Verlauf ein Aufholen der BMD-Werte [5]. Die quantitative Computertomografie (QCT) erfasst die tatsächliche Knochendichte $\left(\mathrm{g} / \mathrm{cm}^{3}\right)$, allerdings ist die Strahlenexposition bei Ermittlung der Daten im Bereich der Wirbelsäule deutlich höher (70-400 $\mu$ Sv). Im Kindesalter hat sich die periphere QCT (pQCT) im Bereich des ultradistalen Radius durchgesetzt. Für Frühund Neugeborene gibt es bisher wenige Daten für die pQCT an Radius und Tibia [6]. Bei männlichen Frühgeborenen konnte im Vergleich zu Reifgeborenen noch in einem Alter von 7 Jahren eine verminderte kortikale Fläche und Dicke im Bereich der Tibia aufgezeigt werden [7]. Die pQCT hat eine hohe Präzision, die bei Kindern mit einem Variationskoeffizienten von 0,8-2,5\% angegeben wird [8]. Nachteil der pQCT ist neben der Strahlenexposition (ca. 1-5 $\mu \mathrm{Sv}$ ) die mangelnde Verfügbarkeit und der hohe apparative Aufwand, der einen Einsatz bspw. in der Neonatologie deutlich einschränkt. Ein wesentlicher Vorteil quantitativer Ultraschallmethoden (QUS) ist neben den geringen Kosten die Möglichkeit, diese auch am Patientenbett bzw. im Inkubator durchzuführen. Nachteile sind neben dem personellen Aufwand und der reduzierten Standardisierung der Messmethoden noch fehlende bzw. unzureichende regionale Referenzwertstudien. Bei üblichen Schallfrequenzen von 0,2 bis $1,2 \mathrm{MHz}$ werden Quer- und Längstransmissionsverfahren unter- schieden, bei denen sich die Ultraschallwellen vom Sender zum Empfänger ausbreiten. Erhobene Werte sind die Abschwächung der Ultraschallwellen (Broadband ultrasound attenuation, BUA in $\mathrm{dB} / \mathrm{MHz}$ ) und die Schallleitungsgeschwindigkeit (Speed of sound, SOS in m/s) bei Durchdringung des Knochens (z. B. am Calcaneus) bzw. Ausbreitung in der Kortikalis des Knochens (z. B. an Radius, Tibia, Phalangen)[9]. Die Messwerte sind von der Knochenmasse, der Knochen-Geometrie und der Knochenelastizität abhängig und korrelieren mit physikalischer Knochendichte, Stabilität der trabekulären Substanz und anderen stofflichen Eigenschaften wie der Elastizität des Knochens. Je dichter bzw. vernetzter eine Struktur ist, umso schneller wird sie von Schallwellen durchdrungen, desto höher ist die SOS. Der QUS stellt mittlerweile bei Erwachsenen und auch im Kindesalter eine etablierte Methode in der Osteoporosediagnostik dar. In den letzten Jahren gibt es auch eine Vielzahl von Studien zum QUS bei Früh- und Neugeborenen, die fast immer eine Korrelation der QUS-Parameter mit dem Gestationsalter zeigten $[10,11]$.

Die vorliegende prospektiv monozentrische Studie bei kaukasischen Neugeborenen hatte folgende Ziele. Für die Bestimmung der SOS sollten verschiedene Messorte (Humerus, Radius, Tibia) evaluiert und die Präzision des verwendeten Sonometers bestimmt werden. Regionale Referenzdaten für die SOS an der Tibia sollten erhoben und Einflussfaktoren auf die SOS untersucht werden. Zudem wurde die praktische Anwendbarkeit der Methode bei Frühund Reifgeborenen in der Neonatologie beurteilt.

\section{Probanden und Methodik}

Die Studie wurde von der lokalen Ethikkommission genehmigt, das schriftliche Einverständnis mindestens eines Elternteils wurde vor Untersuchungsbeginn eingeholt. Ausschlusskriterien für die Studie waren: nicht-kaukasische Herkunft, Knochen- sowie Stoffwechselerkrankungen, Neuromuskuläre Pathologie, renale und endokrine, syndromale und genetische Erkrankungen, schwere ZNS-Pathologie, angeborene Infektionen sowie eine prä-/postnatale Behandlung mit Glukokortikoiden und eine mütterliche Osteomalazie.

Es wurden 219 reife Neugeborene (107 weiblich, 112 männlich) und 14 Frühgeborene ( 6 weiblich, 8 männlich) in die Studie eingeschlossen. An neonatalen Daten wurden erfasst: Geburtsdatum, errechneter/korrigierter Geburtstermin, Geschlecht, Gestationsalter, Postnatales Alter, Geburts- und aktuelles Gewicht, Körperlänge, Kopf- und Brustumfang bei Geburt, $\operatorname{APGAR}(1,5,10)$, NA$\mathrm{pH}, \mathrm{NV}-\mathrm{pH}$, Einling/Mehrling, Geburtsmodus, Medikation, Krankheiten/Infektionen. Zur Abschätzung pränataler Risikofaktoren wurden maternal erfasst: Geburtsdatum, Anzahl Schwangerschaften, Geburten, Tokolyse, Adipositas, Prä-/Eklampsie, Diabetes mellitus/Gestationsdiabetes, Nikotinabusus, Medikation während der Schwangerschaft. Für die Erhebung der Referenzdaten wurden Neugeborene mit folgenden Kriterien ausgeschlossen: Hypo-bzw. Hypertrophie (Geburtsgewicht< 10. bzw. > 90. Perzentile) und mütterlicher Gestationsdiabetes. Die Unterteilung in Früh- bzw. Reifgeborene erfolgte anhand des Gestationsalters (vollendete 37. SSW). Das Gestationsalter lag zwischen $28+3$ und $41+5$ Schwangerschaftswochen (SSW; Mittelwert 39 $\pm 1,8$ SSW, Median 39SSW). Das Geburtsgewicht lag zwischen 590 bis $4930 \mathrm{~g}$ (Mittelwert $3363,5 \pm 557,4 \mathrm{~g}$, Median $3360 \mathrm{~g})$. Die Untersuchung erfolgte in- 
nerhalb der ersten Lebenswoche bei den Reifgeborenen (Mittelwert 1,8 \pm 1,3 Tage, Median 2 Tage) und innerhalb der ersten 11 Lebenstage (Mittelwert 5,1 $\pm 3,5$ Tage, Median 4 Tage) bei den Frühgeborenen und wurde immer von ein und derselben Person durchgeführt.

Die Messungen wurden mit dem Sonometer Sunlight Omnisense 7000 Ртм (Sunlight Med. Ltd. Tel Aviv, Israel) unter Verwendung der für Neugeborene geeigneten kleinsten Ultraschallsonde (CS cortex small) durchgeführt. Erhoben wurde die SOS in Längstransmission im kortikalen Knochen. Die Messungen wurden entsprechend der Vorgaben des Herstellers an der Tibia, aber auch am Humerus und am Radius in Schaftmitte durchgeführt ( $\bullet$ Abb. 1a, b). Die Ankopplung erfolgte durch standardisiert angewärmtes, herkömmliches Ultraschallgel. Die vom Sender des Sonometers abgegebene Ultraschallwelle breitet sich mit einer Frequenz von 1,25 $(0,5-2) \mathrm{MHz}$ unter der Knochenoberfläche bis zum Empfänger aus. Die SOS wird durch Leitung im umgebenden Weichteilgewebe, dem durchschnittlichen Abstand zwischen Sender und Empfänger sowie dem Winkel zwischen Knochenoberfläche und Transducer beeinflusst. Mithilfe der patentierten Omnipath ${ }^{\mathrm{TM}}$-Technologie im Sonometer werden die beeinflussenden Effekte des Weichteilgewebes eliminiert.

Nach Einschalten des Gerätes erfolgte vor Beginn der Untersuchung eine Qualitätsprüfung mittels Phantom (Phantom Perspex, Se-Nr. 522) aus homogenem Hartpolymer, das die Ultraschallwel-
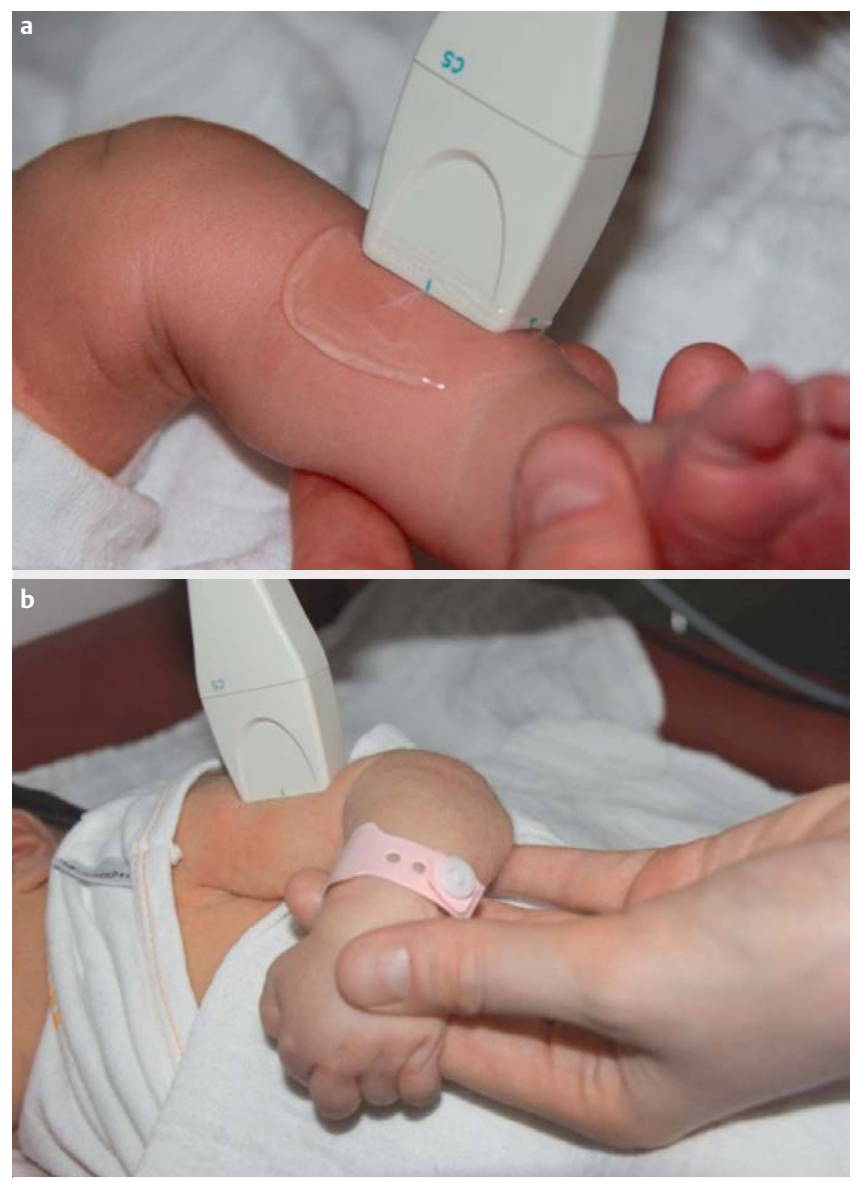

- Abb. 1 a Quantitativer Ultraschall (QUS) an der Tibia, b QUS am Humerus. len in Abhängigkeit von der Umgebungstemperatur in bekannter Geschwindigkeit leitet. Die Langzeitpräzision in vitro wurde durch den Variationskoeffizienten dieser Messungen über den gesamten Zeitraum ermittelt. Die Kurzzeitpräzision wurde bei 30 Neugeborenen mit je 2 Untersuchungsabläufen überprüft. Im Zeitraum von 5-10 Min erfolgten hierzu 2 Messungen am gleichen Ort. Der Variationskoeffizient dieser Messungen wurde erfasst.

Die Probanden-Messungen erfolgten nach Vorgabe des Herstellers standardisiert an der linken Tibia. Zunächst wurde die Strecke zwischen Ferse und Oberseite Patella mittels Maßband ermittelt und halbiert. Der Meßort wurde auf der anterioren Seite der Tibia quer zur Längsachse mit weißem Stift markiert und reichlich Ultraschallgel aufgetragen. Mit dem zentralen Markierungspunkt wurde die CS-Sonde auf der Linie aufgesetzt und nach medial und lateral abgefahren. Bei ordnungsgemäßer Messung ertönt ein akustisches Signal. Zur Erhebung der SOS waren jeweils mindestens 3 bis maximal 5 Messzyklen erforderlich. Zur genaueren Präzision und Vermeidung zufälliger Fehler erfolgten bei jedem Probanden mindestens 2 Messungen, aus denen der Mittelwert gebildet wurde; bei Abweichungen $>1 \%$ (ca. $30 \mathrm{~m} / \mathrm{s}$ ) wurde eine dritte Messung ergänzt. Die Gesamtdauer der Untersuchung von Markierung bis zu drei Messungen lag im Bereich von maximal 10 Min pro Proband.

Um Referenzdaten von verschiedenen Orten zu erheben, wurden exemplarisch Messungen bei 10 Probanden am Humerus und am Radius durchgeführt. Bei keinem dieser Reifgeborenen war es allerdings möglich, ein sonografisch ausreichend stabiles Signal zu erhalten.

Die Daten wurden im Programm Excel 97 (Microsoft Inc, Seattle, WA. USA) erfasst. Die statistische Analyse erfolgte unter Anwendung der Korrelationsanalyse mit SPSS für Windows (Version 20.0, SPSS Inc., Chicago, IL USA). Erhoben wurden Mittelwerte, Median, Varianz, Standardabweichung sowie Minimum und Maximum. Bei der Normwertbestimmung wurde das $95 \%$-Konfidenzintervall dargestellt. Die Daten wurden auf Normalverteilung geprüft. Zum Vergleich der Ergebnisse der QUS-Parameter zwischen den Gruppen wurde der nichtparametrische Mann-Whitney-U-Test und tTests für gepaarte Stichproben durchgeführt. P-Werte von $<0,05$ wurden als statistisch signifikant gewertet. Mittels einfaktorieller ANOVA wurden Einflussfaktoren auf die SOS ermittelt. Die multivariate Analyse des Zusammenhangs von SOS und Variablen erfolgte mittels linearer Regressionsanalyse. Die Präzisionsbestimmung wurde über den Variationskoeffizienten errechnet (VK \% =SD/ $M W x 100)$. Die in vivo Präzision erfolgte nach Empfehlung von Gluer et al. [12].

\section{Ergebnisse}

Der über die gesamte Studienzeit ermittelte Variationskoeffizient der täglichen Qualitätskontrolle mittels Phantom betrug 0,26\% (Mittelwert $2752 \mathrm{~m} / \mathrm{s}$, SD 7,29 m/s). Damit ergab sich eine sehr gute in vitro Langzeitpräzision.

Die Kurzzeitpräzision in vivo lag bei den 30 untersuchten Neugeborenen mit Doppelmessungen bei 0,39\% (Mittelwert $3041 \mathrm{~m} / \mathrm{s}$, SD $11,9 \mathrm{~m} / \mathrm{s}$ ).

Nachdem die Probemessungen an Radius und Humerus bei jeweils 10 Probanden nicht zu reproduzierbaren Ergebnissen führten bzw. nicht erfolgreich waren, wurde die Studie nur an der linken 
Tibia durchgeführt. Bei insgesamt 232 Probanden waren die Messungen erfolgreich ( $\triangleright$ Tab. 1). Bei einem hypertrophen männlichen Probanden konnte trotz mehrfacher Versuche kein ausreichendes Ultraschallsignal abgeleitet werden. Bei den Reifgeborenen fielen signifikante Unterschiede zwischen männlichen und weiblichen Probanden auf. Männliche Reifgeborene hatten ein signifikant höheres Geburtsgewicht $(3497,5 \pm 465,3 \mathrm{~g})$ als weibliche $(3359,9 \pm 461,4 \mathrm{~g} ; \mathrm{p}=0,03$ und einen signifikant größeren Kopfumfang $(34,9 \pm 1,5$ vs. $34,2 \pm 1,5 \mathrm{~cm} ; \mathrm{p}=0,001)$, während Gestationsalter, Geburtslänge und Brustumfang nicht signifikant verschieden waren.

Für die Referenzdatenbestimmung wurden insgesamt 178 Probanden (85 weiblich, 93 männlich) ausgewertet ( $>$ Tab. 2). Die erhobenen SOS-Mittelwerte zeigten bezogen auf das Gestationsalter und das Geburtsgewicht eine signifikante Differenz der SOS-Werte (p 0,04).

Mit zunehmendem Gestationsalter konnte von der 37. bis zur 41. SSW bei einem Korrelationskoeffizient von 0,22 ein signifikanter Anstieg der SOS von $3012 \pm 87$ bis $3069 \pm 102 \mathrm{~m} / \mathrm{s}$ gezeigt werden $(p=0,003)$. Die Beziehung zwischen dem Geburtsgewicht von 2550 bis $4150 \mathrm{~g}$ und SOS in der Referenzpopulation ergab keine signifikante Korrelation ( $\$$ Abb. 2a und b). In Abhängigkeit vom Geschlecht zeigten in der Referenzgruppe männliche Neugeborene signifikant höhere Werte ( $p=0,012 ;$ > Tab. 3).

- Tab. 1 Probandencharakteristik in Bezug zum Gestationsalter.

\begin{tabular}{|l|l|l|}
\hline & $\begin{array}{l}\text { Reifgeborene } \\
\geq 37 \text { SSW }\end{array}$ & $\begin{array}{l}\text { Frühgeborene } \\
<37 \text { SSW }\end{array}$ \\
\hline Anzahl & 219 & 14 \\
\hline Weiblich/Männlich & $107 / 112$ & $6 / 8$ \\
\hline Einling/Zwilling & $216 / 3$ & $12 / 2$ \\
\hline Gestationsalter in SSW & $37+1-41+5$ & $28+3-36+6$ \\
\hline (MW \pm SD) & $39,3 \pm 1,2$ & $33,9 \pm 2,7$ \\
\hline Geburtsgewicht in g & $2410-4930$ & $590-3400$ \\
\hline (MW \pm SD) & $3430,3 \pm 467,4$ & $2319,3 \pm 794,3$ \\
\hline Geburtslänge in cm & $45-59$ & $31-50$ \\
\hline (MW \pm SD) & $50,9 \pm 2,4$ & $43,5 \pm 6,1$ \\
\hline Kopfumfang in cm & $31-39$ & $23-37$ \\
\hline (MW \pm SD) & $34,6 \pm 1,5$ & $31,4 \pm 4,8$ \\
\hline & & \\
\hline
\end{tabular}

Bei hypotrophen Reifgeborenen (Geburtsgewicht $<10$. Perzentile, $n=20$ ) bewegten sich die SOS bei 3047 bis $3088 \mathrm{~m} / \mathrm{s}$ und lagen mit Ausnahme der 41. SSW stets oberhalb der Messwerte normotropher Reifgeborener $(n=178)$. Der Unterschied der gruppierten SOS-Mittelwerte $(3068 \pm 90 \mathrm{~cm} / \mathrm{s})$ zu eutrophen Neugeborenen $(3039 \pm 99 \mathrm{~cm} / \mathrm{s})$ war nach dem Mann-Whitney-U-Test signifikant $(p<0,001)$. Bei hypertrophen Reifgeborenen (Geburtsgewicht $>90$. Perzentile, $n=19$ ) wurden SOS-Mittelwerte von 2944 bis $3070 \mathrm{~m} / \mathrm{s}$ ermittelt. Im Gruppenvergleich lagen die SOS-Mittelwerte hypertropher (3016 $\pm 83 \mathrm{~ms} /$ ) unterhalb der von eutrophen Neugeborenen (p 0,015)( $\triangleright$ Abb. 3). Für die untersuchten 14 Frühgeborenen wurde eine SOS von $2991 \pm 118 \mathrm{~cm} / \mathrm{s}$ ermittelt. Unter Berücksichtigung der Daten für die eutrophen Neugeborenen ergab sich ein signifikanter Unterschied der SOS an der Tibia $(p<0,05)$. Auch im Direktvergleich der SOS-Mittelwerte aller Früh- und aller Reifgeborenen war der Unterschied statistisch signifikant $(p<0,05)$. Kein statistisch signifikanter Einfluss auf die SOS konnte für die mütterlichen (Gestationsdiabetes, Nikotinabusus, Adipositas, Medikation, Alter, Erkrankungen) und für die kindlichen Faktoren (Geburtsmodus, Größenparameter, Einling/Zwilling) bestätigt werden. In der Regressionsanalyse wurden für die SOS an der Tibia das Gestationsalter und Geschlecht als unabhängige Prognosefaktoren nachgewiesen.

\section{Diskussion}

Der quantitative Ultraschall (QUS) ist eine adäquate Methode zur Beurteilung der Knochenmineralisation und Knochenstruktur des peripheren Skeletts. Einfluss auf die erhobenen Parameter haben die mechanischen Eigenschaften des Knochens wie Mineraldichte, Kortikalisdicke und Elastizität. Vorteilhaft sind fehlende ionisierende Strahlung, Mobilität mit teilweise möglicher bettseitiger Diagnostik, kurze Untersuchungsdauer. Bevor eine Untersuchungsmethode in der Routine verwendet wird, sind Präzision und Reproduzierbarkeit sowie Referenzdaten notwendig. Im Kindesalter wird der QUS im klinischen Alltag aufgrund begrenzter methodischer Reproduzierbarkeit, relativer Messungenauigkeiten und Varianz sowie mangelnder Validierung der unterschiedlichen Geräte an verschiedenen Messorten noch nicht regelmäßig eingesetzt [13]. Zumeist liegen nur unzureichend Referenzwertdaten vor, die aber Grundvoraussetzung für Patientenstudien sind. Die vorliegende Studie stellt nach unserer Kenntnis die größte regionale ReferenzUntersuchung an kaukasischen Neugeborenen dar und liefert Aussagen zur Präzision unter Anwendung einer standardisierten Untersuchungstechnik.

- Tab. 2 SOS Referenzwerte mit 95\%-Referenzbereich bei eutrophen Neugeborenen.

\begin{tabular}{|c|c|c|c|c|c|}
\hline \multirow[t]{2}{*}{$\begin{array}{l}\text { Gestationsalter } \\
\text { (SSW) }\end{array}$} & \multirow[t]{2}{*}{$n$} & \multirow[t]{2}{*}{$\begin{array}{c}\text { Geburtsgewicht } \\
\text { (g) } \pm \text { SD }\end{array}$} & \multirow[t]{2}{*}{$\begin{array}{l}\text { SOS-MW } \\
(\mathrm{m} / \mathrm{s}) \pm \text { SD }\end{array}$} & \multicolumn{2}{|c|}{$\begin{array}{l}95 \% \text { Referenzbereich } \\
\text { für SOS-MW }(\mathrm{m} / \mathrm{s})\end{array}$} \\
\hline & & & & Untergrenze & Obergrenze \\
\hline $37+0-39+6$ & 93 & $3271 \pm 307$ & $3028 \pm 92$ & 3009 & 3047 \\
\hline $40+0-41+6$ & 85 & $3581 \pm 301$ & $3057 \pm 96$ & 3036 & 3077 \\
\hline Gesamt & 178 & $3419 \pm 341$ & $3041 \pm 95$ & 3027 & 3055 \\
\hline
\end{tabular}




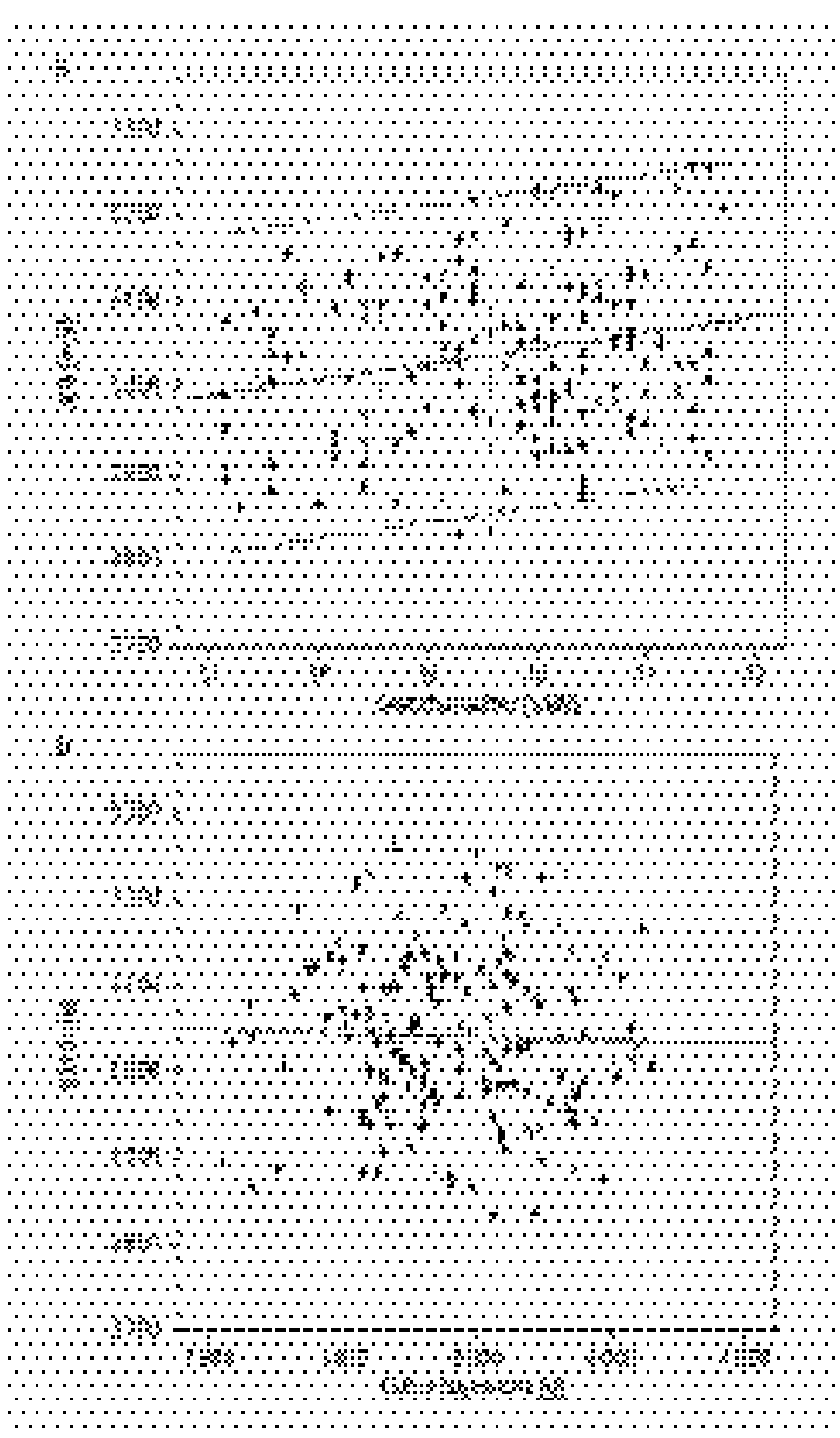

- Abb. 2 a SOS (Speed of Sound, Schallleitungsgeschwindigkeit im kortikalen Knochen) und Gestationsalter b SOS und Geburtsgewicht.

Die ermittelte in vitro Langzeitpräzision des verwendeten Sonometers von 0,26 \% für die Phantomuntersuchungen über den gesamten Untersuchungszeitraum von 22 Monaten liegt im Bereich bisher dazu publizierter Daten, die von 0,10 bis $0,63 \%$ reichen [14]. Der Forderung nach Phantomen, die einen knochenähnlichen Aufbau zeigen und eine Kreuzkalibrierung und somit Vergleichbarkeit verschiedener Gerätetypen ermöglichen, wurde bislang nicht entsprochen [13]. Die Kurzzeitpräzision in vivo mit einem Variationskoeffizienten von 0,39\% bei Neugeborenen liegt im Bereich über das gesamte Kindesalter publizierter $0,2-0,4 \%$ von Zadik et al. [15], aber deutlich besser als die Messungen von Fewtrell et al. bei Frühgeborenen mit Werten von 1-2\% [16] und von Liao et al. bei Früh- und Neugeborenen mit Werten von 1,23-1,87\% [17]. Die Untersucherabhängigkeit der in vivo Präzision wurde aktuell nicht untersucht. Nach Pereira-da-Silva et al. liegt sie bei 1,2\% [18].

Die Bedeutung lokaler Referenzwertstudien ist bekannt, so erhoben Liao et al. an einem großen asiatischen Kollektiv von 267 Neu- geborenen deutlich niedrigere SOS-Werte als bei kaukasischen Kindern publiziert wurden [19]. Das bislang größte publizierte asiatische Kollektiv erfasst 667 Kinder ( 370 weiblich, 297 männlich), davon 338 vor der 37. SSW und 329 nach vollendeter 37. SSW geboren [20]. Bei den Reifgeborenen wurden - wie in der vorliegenden Studie - signifikant höhere SOS an der Tibia gemessen als bei den Frühgeborenen ( $2971,7 \pm 106,3 \mathrm{~m} / \mathrm{s}$ vs. $2932,9 \pm 112,4 \mathrm{~m} / \mathrm{s})$, als Einflussfaktoren bei Reifgeborenen konnten das Gestationsalter und das Geburtsgewicht ermittelt werden. Zuccotti et al. [21] beobachteten in ihrer Studie keinen signifikanten Zusammenhang zwischen SOS und dem Gestationsalter, dem Gewicht, der Körperlänge und dem Kopfumfang. Sie führen das darauf zurück, dass sie nur Reifgeborene untersucht haben, während in den anderen Studien auch Frühgeborene einbezogen wurden. In ihrer Longitudinalstudie bis zum vollendeten 12. Lebensmonat stellten sie nach einer initialen Abnahme der SOS (nicht signifikant) in den ersten 4 Monaten einen deutlichen Anstieg der SOS an der Tibia bis zum ersten Geburtstag fest. Bei der klinischen Beurteilung und Wertung von QUS-Parametern in der Neugeborenen- und Säuglingszeit ist ein Bezug zum jeweiligen Alter des Kindes sehr wichtig.

In der aktuellen Referenzpopulation wurde bei eutrophen kaukasischen Reifgeborenen eine Geschlechtsabhängigkeit mit signifikant höherer SOS für die männliche Gruppe (3058 \pm 93 vs. $3023 \pm$ $93 \mathrm{~m} / \mathrm{s}$ ) ermittelt. Die Studie von Zuccotti et al. mit 62 männlichen und 54 weiblichen Neugeborenen zeigte Werte von 3042 bzw. $2964 \mathrm{~m} / \mathrm{s}$, allerdings ohne statistische Signifikanz [21]. Teitelbaum et al. [22] beschreiben einen SOS-Mittelwert von $3012 \mathrm{~m} / \mathrm{s}$ über alle untersuchte Reifgeborene, Mc Devitt et al. [10] von $3079 \mathrm{~m} / \mathrm{s}$, Yiallourides et al. [23] von $3100 \mathrm{~m} / \mathrm{s}$ und Schushan-Eisen et al. [24] $3120 \mathrm{~m} / \mathrm{s}$.

Der Zusammenhang zwischen Geburtsgewicht und SOS der Tibia nahm in der untersuchten Gruppe mit zunehmendem Reifegrad ab. Wurden nur die eutrophen Früh-/Neugeborenen bis zu maximal $3800 \mathrm{~g}(n=165)$ betrachtet, so bestand eine signifikante positive Korrelation $(p=0,009)$. Unter Berücksichtigung des gesamten Kollektivs, zeigt sich keine signifikante Korrelation. Diese Ergebnisse stimmen mit Zuccotti et al. überein [21]. Die von Chen et al. [25] beobachtete negative Korrelation (hohes Geburtsgewicht - geringere SOS) könnte darauf zurückzuführen sein, dass hypertrophe Feten infolge von Platzmangel und verminderter Bewegungsfreiheit eine niedrigere Knochenstabilität (Mineralisation, Dichte, Elastizität) aufweisen. Auch Littner et al. zeigten bei hypertrophen Neugeborenen von Müttern mit Gestationsdiabetes signifikant niedrigere SOS-Werte [26], während die bei uns beobachtet höheren SOS-Werte von hypotrophen Neugeborenen im Vergleich zu eutrophen von Koo et al. bestätigt werden [27]. Auch wenn bei dem in unserer Studie eingesetzten Sonometer seitens des Geräteherstellers durch Anwendung der Omnipath ${ }^{\mathrm{TM}}$ Technologie ein Weichteileffekt negiert wird, sollte dieser Effekt zumindest kritisch hinterfragt werden.

Der Versuch, bei Früh- und Reifgeborenen unter Beibehaltung des Sonometers und CS-Schallkopfes verschiedene Messorte zur Erhebung quantitativer Ultraschallparameter der größeren kortikalen Röhrenknochen mittels Längstransmission zu etablieren, war - wie in der Literatur bestätigt - leider auch in unserer Studie nicht erfolgreich [28]. Wir führen das auf die erschwerte Durchführung der Messungen an Radius und Humerus mit mangelnder Fixierbar- 
- Tab. 3 Geschlechtsbezogener Vergleich von SOS und somatischen Parametern als Referenzdaten der Reifgeborenen.

\begin{tabular}{|l|l|l|}
\hline $\begin{array}{l}\text { Parameter } \\
\text { MW } \pm \text { SD }\end{array}$ & Weiblich (n=85) & Männlich (n=93) \\
\hline SOS (m/s) & $3022,8 \pm 93,4$ & $3058,3 \pm 93,3$ \\
\hline Gestationsalter (SSW) & $39,7 \pm 1,1$ & $39,7 \pm 1,21$ \\
\hline Geburtsgewicht (g) & $3369,8 \pm 338,8$ & $3464,2 \pm 338,1$ \\
\hline Geburtslänge $(\mathrm{cm})$ & $50,6 \pm 2,0$ & $50,9 \pm 2,1$ \\
\hline Kopfumfang (cm) & $34,4 \pm 1,4$ & $34,8 \pm 1,4$ \\
\hline Brustumfang $(\mathrm{cm})$ & $33,4 \pm 1,7$ & 0,06 \\
\hline
\end{tabular}

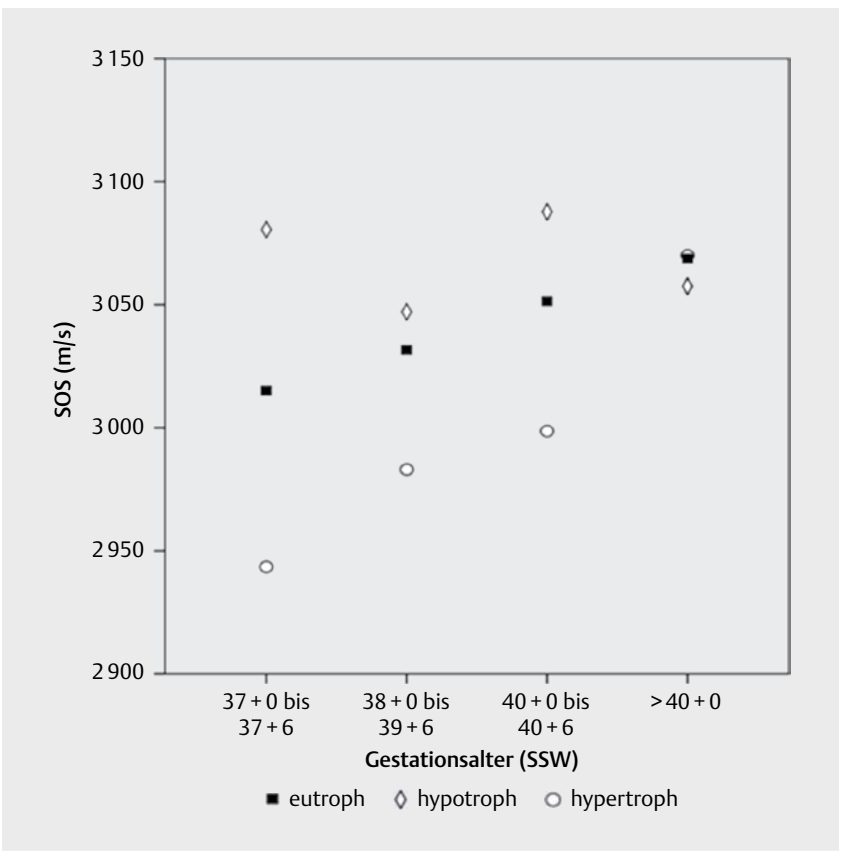

Abb. 3 Beziehung von SOS (Speed of sound) und Gestationsalter (SSW - Schwangerschaftswochen) in Abhängigkeit vom Gewicht des Neugeborenen. nen Messorte (trabekulär versus kortikaler Knochen). Limitiert sind die Daten in ihrer Aussagekraft bei den Frühgeborenen in der geringen Gruppengröße sowie darin, dass der mögliche positive Effekt einer Physiotherapie nicht erfasst wurde.

Zusammenfassend konnte in dieser deutschen Referenzwertstudie ein signifikanter Zusammenhang zwischen Geburtsalter und Schallgeschwindigkeit (SOS) erhoben werden. Männliche Neugeborene wiesen signifikant höhere Werte auf. Die untersuchten Frühgeborenen zeigten signifikant niedrigere SOS-Werte.

\section{Kernaussagen}

Die standardisierte Untersuchung mittels Omnisense 7000 P unter Verwendung der kleinen CS-Sonde zeichnet sich durch hohe in vitro und in vivo Präzision aus. Als Messort für die SOS-Erfassung ist bei Früh- und Reifgeborenen nur die Tibia geeignet und gut praktikabel in der Neonatologie anwendbar. Bei dem regionalen kaukasischen Referenzkollektiv konnten QUS-Daten für Neugeborene erhoben werden, die für nachfolgende Studien bei Frühgeborenen und zur Beurteilung der Osteopenie genutzt werden können, wobei Geschlecht und Trophik zu beachten sind. Zwischen SOS und Gestationsalter besteht ein signifikanter positiver Zusammenhang. Bezogen auf das Geschlecht besteht ein Zusammenhang zur SOS mit höheren Werten bei männlichen Probanden.

\section{Interessenkonflikt}

Die Autoren geben an, dass kein Interessenkonflikt besteht.

\section{Literatur}

[1] Rauch F, Schönau E. Skeletal development in premature infants: a review of bone physiology beyond nutritional aspects. Arch Dis Child Fetal Neonatal 2002; 86: F82-F85

[2] Rigo ], Pieltain C, Salle B et al. Enteral calcium, phosphate and vitamin $D$ requirements and bone mineralisation in preterm infants. Acta Paediatr 2007; 96: 969-974

[3] Rack B, Lochmüller EM, Janni W et al. Ultrasound for the assessment of bone quality in preterm and term infants. J Perinatol 2012; 32: 218-226 
[4] http://www.lutherhaus.de/osteo/leitlinien-dvo

[5] Quintal VS, Diniz EM, Caparbo VF et al. Bone densitometry by dual-energy $X$-ray absorptiometry (DXA) in preterm newborns compared with full-term peers in the first six months of life. J Pediatr (Rio J) 2014; 90: 556-562

[6] Moyer-Mileur L], Quick JL, Murray MA. Peripheral quantitative computed tomography of the tibia: pediatric reference values. J Clin Densitom 2008; 11: 283-294

[7] Abou Samra H, Stevens D, Binkley T et al. Determinants of bone mass and size in 7-old-former term, late-preterm, and preterm boys. Osteoporos Int 2009; 20: 1903-1910

[8] Gilsanz V. Bone density in children: a review of the available techniques and indications. Eur J Radiol 1998; 26: 177-182

[9] Mora S, Vigano A, Cafarelli L et al. Applicability of quantitative ultrasonography oft he radius and tibia in HIV-infected children and adolescents. J Acquir Immune Defic Syndr 2009; 51: 588-592

[10] Mc Devitt H, Ahmed SF. Quantitative ultrasound assessment of bone health in the neonate. Neonatology 2007; 91: 2-11

[11] Rack B, Lochmüller EM, Janni W et al. Ultrasound for the assessment of bone quality in preterm and term infants. J Perinatol 2012; 32: 218-226

[12] Gluer CC, Blake G, Lu Y et al. Accurate assessment of precision errors: how to measure the reproducibility of bone densitometry techniques. Osteoporos Int 1995; 5: 262-270

[13] Mohr A, Barkman R, Mohr C et al. Quantitative ultrasound for the diagnosis of osteoporosis. RöFo 2004; 176: 610-617

[14] Knapp KM, Blake GM, Spector TD et al. Multisite quantitative ultrasound: precision, age- and menopause-related changes, fracture discrimination, and T-score equivalence with dual-energy X-ray absorptiometry. Osteoporos Int 2001; 12: 456-464

[15] Zadik Z, Price D, Diamond G. Pediatric reference curves for multi-site quantitative ultrasound and its modulators. Osteoporos Int 2003; 14: 857-862

[16] Fewtrell MS, Loh KL, Chomtho S et al. Quantitative ultrasound (QUS): a useful tool for monitoring bone health in preterm infant? Actra Paediatr 2008; 97: 1625-1630

[17] Liao XP, Zhang WL, He J et al. Bone measurements of infants in the first 3 months of life by quantitative ultrasound: the influence of gestational age, season, and postnatal age. Pediatr Radiol 2005; 35 : 847-853
[18] Pereira-da-Silva L, Costa A, Pereira L et al. Early high calcium and phosphorus intake by parenteral nutrition prevents short-term bone strength decline in preterm infants. J Pediatr Gastroenterol Nutr 2011; 52: 203-209

[19] Liao XP, Zhang WL, Yan CH et al. Reduced tibial speed of sound in Chinese infants at birth compared with Caucasian peers: the effects of race, gender, and vitamin $\mathrm{D}$ on fetal bone development. Osteoporos Int 2010; 21: 2003-2011

[20] Chen HL, Tseng HI, Yang RC. Bone status and associated factores measured by quantitative ultrasound in preterm and full-term newborn infants. Early Hum Devel 2012; 88: 617-622

[21] Zuccotti G, Vigano A, Cafarelli L et al. Longitudinal changes of bone ultrasound measurements in healthy infants during the first year of life: influence of gender and type of feeding. Calcif Tissue Int 2011; 89: 312-317

[22] Teitelbaum JE, Rodriquez RJ, Ashmeade TL et al. Quantitative ultrasound in the evaluation of bone status in premature and full-term infants. J Clin Densitom 2006; 9: 358-362

[23] Yiallourides M, Savoia M, May J et al. Tibial speed of sound in term and preterm infants. Biol Neonate 2004; 85: 225-228

[24] Schushan-Eisen I, Cohen M, Leibovitch L et al. Bone density among infants of gestational diabetic mothers and macrosomic neonates. Matern Child Health J 2014; 19: 578-582

[25] Chen HL, Tseng HI, Yang SN et al. Bone status and associated factors measured by quantitative ultrasound in preterm and full-term newborn infants. Early Hum Dev 2012; 88: 617-622

[26] Littner Y, Mandel D, Mimouni FB et al. Decreased bone ultrasound velocity in large-for-gestational-age infants. J Perinatol 2004; 24: 21-23

[27] Koo WW, Bajaj M, Hockman EM et al. Bone ultrasound velocity in neonates with intrauterine growth deficit reflects a growth continuum. J Clin Densitom 2010; 14: 28-32

[28] Koo WW, Bajaj M, Mosely M et al. Quantitative bone US measurements in neonates and their mothers. Pediatr Radiol 2008; 38: 1323-1329

[29] Krikke M, Yumani D, Rustenburg C et al. Assessing bone development in preterm infants using quantitative ultrasonography showed a decline in the early postnatal period. Acta Pediatr 2017; 107: 227-233

[30] Lageweg CMT, van der Putten ME, van Goudoever JB et al. Evaluation of bone mineralisation in former preterm born children: phalangeal quantitative ultrasound cannot replace dual-energy X-ray absorptiometry. Bone Reports 2018; 8: 38-45 